Психология. Журнал Высшей школы экономики,

2021. T. 18. № 3. C. 623-642. DOI: 10.17323/1813-8918-2021-3-623-642

\title{
НЕЙРОФИЗИОЛОГИЧЕСКИЕ МЕХАНИЗМЫ РЕШЕНИЯ ЭКСПЕРИМЕНТАЛЬНЫХ ТВОРЧЕСКИХ ЗАДАЧ: ИНСАЙТ И/ИЛИ КРИТИЧЕСКИЙ АНАЛИЗ?
}

\author{
О.М. РАЗУМНИКОВА
}

\begin{abstract}
${ }^{a}$ Новосибирский государственный технический университет, 630073, Россия, Новосибирск, пр. К. Маркса, д. 20
\end{abstract}

\section{Резюме}

Рассмотрены разные точки зрения на процессы взаимодействия нейронных сетей мозга при селекции информации и принятия решения на основе инсайта или критического анализа идей, сгенерированных при тестировании креативности. Для изучения сочетания разных когнитивных операций в креативности предложены модели BVSR (Blind Variation and Selective Retention) и «GENEPLORE» (Generate and Explore). Анализ возникновения инсайта в экспериментальных исследованиях выполняется с использованием задач на эвристическое мышление, восприятие двойственных фигур, теста отдаленных ассоциаций слов (RAT) или составных отдаленных ассоциаций (CRA). Приведены сведения о наиболее устойчивом ЭЭГ-корреляте инсайта: синхронизации альфа-биопотенциалов и изменениях выраженности и регионарной специфики этого эффекта, обусловленных особенностями креативного задания. Результаты томографических исследований выявили ключевое значение специфичных паттернов «преднастройки» нейронных сетей дефолтной и исполнительной систем и динамики взаимодействия лобных и теменных областей мозга, отражающих разные показатели креативности. Показаны роль критического анализа решения проблемы, модулирующая творческий процесс, и значение тормозных функций в селекции релевантной информации. Включение в поиск решения проблемы противоположных процессов - конвергентного или дивергентного мышления, направленного или дефокусированного и интернального или экстернального внимания - сопровождается временной и структурной реорганизацией активации или торможения в нейронных системах мозга. Ведущими структурами мозга, необходимыми для переключения разных стратегий селекции информации и выбора ответа при решении экспериментальных творческих задач в зависимости от их сложности и содержания, являются префронтальная, цингулярная области коры и гиппокамп.

Ключевые слова: креативность, инсайт, критическое мышление, селекция информации, тормозные функции, нейронные сети, электроэнцефалография, томография.

Развитие технических средств регистрации активности мозга и математических методов анализа этих данных стало основой интенсивных исследований закономерностей процессов мышления, в том числе творческого мышления

Работа выполнена при поддержке Российского фонда фундаментальных исследований (проект № 19-29-001017). 
(Beaty et al., 2016, 2018; Benedek et al., 2014; Khalil et al., 2019; Dandan et al., 2013). При этом выделяются два принципиально разных пути поиска новых решений: реконструкция восприятия проблемы (т.е. увидеть ее в новом свете) и концептуальная реконструкция (разные формы понимания ситуации). Изучение инсайта - спонтанного решения проблем разного характера, не только решения задач при тестировании креативности, - вызывает особый интерес как способность внезапного ясного понимания проблемы или ситуации. Согласно одной из моделей креативности, спонтанность и неосознанность формирования инсайта наряду со стратегией планирования рассматриваются как разные режимы креативного мышления (Dietrich, 2004; Dietrich, Kanso, 2010). Интерес к пониманию нейробиологии инсайта подчеркивается резко возросшим числом публикаций по этой теме: 771 за последние 5 лет, согласно базе PubMed. Однако обзор литературы, посвященный выяснению нейробиологических основ инсайта, выявил «противоречивость и гетерогенность результатов проведенных исследований» (Бакулин и др., 2020, с. 81), вследствие чего сделано заключение о необходимости дальнейшего продолжения междисциплинарных исследований инсайта.

В экспериментальных условиях выяснения закономерностей возникновения инсайта обычно применяется самоотчет об использованной стратегии решения поставленной задачи, наряду с инсайтом задействуется последовательный перебор спонтанно или целенаправленно возникающих идей и выбор наиболее подходящей. Такая аналитическая стратегия творческого мышления особенно важна для решения научных и технических проблем (Lawson, 2010; Wechsler et al., 2018). Настоящий обзор посвящен описанию нейрофизиологических процессов, связанных с принципиально разными механизмами креативности: инсайтом или аналитическим способом решения экспериментальных творческих задач и соответствующими им разными формами селекции информации.

\section{Нейрофизиологические корреляты инсайта при решении творческой задачи}

Согласно определению инсайта, данному в Психологическом словаре, это «внезапное и невыводимое из прошлого опыта понимание существенных отношений и структуры ситуации в целом, посредством которого достигается осмысленное решение проблемы» (Психологический словарь, 2007, с. 274). Таким образом, основными характеристиками инсайта являются внезапность нахождения решения проблемы и уверенность в том, что это именно то решение, которое требуется. Для анализа возникновения инсайта в экспериментальных исследованиях применяют задачи на эвристическое мышление, восприятие двойственных фигур либо тест отдаленных ассоциаций слов (RAT) или составных отдаленных ассоциаций (CRA) (Бакулин и др., 2020; Шелепин, Шелепин, 2015; Sprugnoli et al., 2017; Webb et al., 2016). RAT используется в нейрофизиологических исследованиях сравнительно чаще других и имеет разнообразные модификации. В связи с тем, что в качестве 
одной из причин противоречивости в нейробиологических коррелятах инсайта отмечено разнообразие используемых методик (Бакулин и др., 2020; Dietrich, Kanso, 2010), остановимся преимущественно на тех результатах исследований инсайта, которые получены с применением RAT, так как эта методика часто используется для изучения нейрофизиологических механизмов инсайта.

RAT включает набор триад слов, к которым необходимо подобрать словоассоциацию (Mednick, 1962; Mednick, Mednick, 1971). В первоначальном варианте теста на поиск ответов для 30 триад отводилось 40 минут, и, согласно полученным результатам, наиболее оригинальные идеи возникали в конце выполнения задания вместе со снижением беглости их генерации. Потенциальное разнообразие возможных ответов позволяет отнести RAT к заданиям, выявляющим способность к дивергентному мышлению. Оригинальность ответов при этом определяется на основе сравнения с имеющейся базой слов-ассоциаций, частота встречаемости которых и определяет степень их стереотипности или новизны (Разумникова, Ларина, 2005; Razumnikova, 2007). Выделено несколько систематических стратегий поиска ассоциаций: догадки возникают либо локально в семантическом пространстве одного из трех слов, либо вследствие его реструктуризации в ходе первых неудачных попыток, либо как эвристика (Fleck, Weisberg, 2013; Smith et al., 2013). Часто RAT рассматривают как тест на конвергентные вербальные способности. Это происходит в том случае, когда конструкция триады слов предусматривает только один правильный ответ: например, при составлении сложных слов из нескольких простых (CAT - Compound Associate Test Kizilirmak et al., 2016; Webb et al., 2016).

B нейрофизиологических исследованиях используют RAT на английском (Beeman, Bowden, 2000), китайском (Qiu et al., 2010), русском (Разумникова, Ларина, 2005; Razumnikova, 2007) и других языках с вариацией количества триад-стимулов от 20 до 192. Рассмотрим несколько ключевых, с нашей точки зрения, работ с применением RAT для исследования вербальной креативности.

Первые нейрофизиологические исследования инсайтной стратегии выполнения RAT показали доминирование правого полушария при воспроизведении необычных ассоциаций (Beeman, Bowden, 2000) и специализацию правой передней части височной извилины при использовании инсайтной стратегии по сравнению с неинсайтной (Jung-Beeman et al., 2004). ЭЭГ-коррелятами инсайта оказались альфа- и гамма-осцилляции: ответу предшествовало повышение мощности альфа-ритма с последующим ростом гамма-биопотенциалов. Сходный эффект локализации вызванной инсайтом нейронной активности в верхней части правой височной извилины был также показан при анализе ЭЭГ-коррелятов другой вербальной задачи - китайских анаграмм (Zhang et al., 2011). Позднее была установлена связь инсайтной стратегии с интернальным вниманием и «грубым» (coarse) правополушарным семантическим кодированием (Kounios, Beeman, 2014). Следует отметить, что для различения инсайтной или неинсайтной стратегий обычно используется самоотчет участников исследования, что внушает опасения в его точности. Однако 
существует точка зрения, что именно такой подход оказывается наиболее приемлемым для экспериментального изучения инсайта (Laukkonen, Tangen, 2018).

Специально организованный эксперимент, в котором испытуемые не только идентифицировали инсайтную стратегию, но и отмечали ответы с генерацией нового решения или узнавания верного ответа среди предъявленных, выявил разную реактивность альфа-ритма (Rothmaler et al., 2017). Так называемому «внутреннему» инсайту предшествовало повышение мощности альфа-колебаний в правой теменной области коры, тогда как «внешнему» (узнаванию ответа) - снижение. Эти разнонаправленные реакции авторы связали с ориентацией на внутренний или внешний фокусы внимания соответственно.

Правополушарной гипотезы генерации инсайта придерживаются авторы фМРТ-исследования с использованием экспериментальной ситуации отгадывания китайских загадок, так как специфичным коррелятом инсайта оказалось усиление функциональной связности между нейронными ансамблями в нижней лобной и средней височной извилинах правого полушария, тогда как билатеральная активация этих областей мозга, а также гиппокампа была общим эффектом вне зависимости от применяемой стратегии решения загадки (Zhao et al., 2014). Вместе с этим на основе оценки энтропии фМРТ-сигнала обнаружена большая левополушарная специализация нижней лобной и средней височной извилин для эффективности дивергентного вербального мышления, что рассматривается авторами исследования как доказательство включения этих структур в регуляцию когнитивной гибкости и тормозный контроль селекции ответов (Shi et al., 2020).

С использованием фМРТ показана связь креативности и регионарной специфичности в структурной организации мозга (Li et al., 2019). Оригинальность ответов при выполнении RAT положительно коррелировала с показателем плотности серого вещества в правой передней верхней височной извилине, но отрицательно - в правой дорзальной части передней цингулярной коры, тогда как с плотностью белого - связь в этой части цингулярной коры была, напротив, положительна, а негативная корреляция отмечена для левой верхней фронтальной извилины. Эти результаты рассматривают как подтверждение модели BAIS (Bilateral Activation, Integration, and Selection) (Jung-Beeman, 2004; Virtue et al., 2008), согласно которой для поиска вербального ответа требуются три семантические функции: семантическая активация, семантическая интеграция и семантическая селекция. Эти функции поддерживаются билатеральной активностью трех участков мозга, расположенных в задней средней и верхней височной извилинах, передней средней и верхней височной и нижней лобной извилинах.

Результаты другого исследования RAT выявили ключевое значение височных областей коры в интеграции активности распределенной нейронной сети, включающей теменную, веретенообразную и угловую извилины, для дискриминации отдаленных и тесно связанных ассоциаций (Shen et al., 2017). Формирование отдаленных ассоциаций сопровождалось повышением 
показателя эффективности связности в узлах, расположенных в перечисленных областях с доминированием правого полушария, тогда как для стереотипных ассоциаций было характерно снижение этого показателя в средней височной извилине.

Поиск оригинального ответа-ассоциации на предложенные стимулы предполагает активацию системы семантически организованной памяти. Исследование нейрофизиологических коррелятов САТ, выполненное на основе фМРТ, выявило, что инсайтное решение сопровождалось активацией ростральной части передней цингулярной коры/медиальной префронтальной коры и левой части гиппокампа (Kizilirmak et al., 2016). Авторы полагают, что такая регионарная специфика активации коры отражает реакцию на новизну сгенерированной идеи и обнаружение новых ассоциаций элементов и новых отношений между ними. Распределенная нейронная система с включением в нее правой префронтальной, передней цингулярной коры и инсулы обнаружена при инсайтном решении анаграмм, сравниваемом с поисковой стратегией, начальный этап которой включает функции зоны Брока и правой инсулы (Aziz-Zadeh et al., 2009). Авторы этого исследования полагают, что функции правой префронтальной и передней цингулярной коры отражают процессы внимания и перебора разных вариантов решения задачи как необходимые для инсайта процессы. Другая широко распределенная система мозговых структур, включающая дефолтную, семантическую и моторную системы, обнаружена с использованием фМРТ и последующим выполнения батареи заданий для тестирования инсайта (Ogawa et al., 2018). Специфическим коррелятом инсайта оказался объем серого вещества в левой части мозжечка и правой добавочной моторной области.

Значение сетей «бодрствующего покоя»: дефолтной нейронной сети и системы исполнительного контроля на разных этапах решения творческих задач - подробно обсуждается в статье Б.М. Величковского с соавт. (Величковский и др., 2019). Взаимодействие этих систем, согласно результатам серии исследований, выполненных Р. Бити с коллегами (Beaty et al., 2014a, 2014b, 2015, 2016, 2019), связано со стадией дивергентного мышления и с индивидуальными способностями к его осуществлению за счет кооперации нейронных сетей дефолт-системы, центральным звеном которой является задняя часть цингулярной коры, и исполнительной системы с функциональным «узлом» в дорзолатеральной префронтальной коре. Такая кооперация предположительно отражает эффекты спонтанного мышления как «преднастройки» к последующей когнитивной деятельности с фокусированием интернального внимания и целенаправленным top-down контролем извлечения информации из памяти с подавлением доминантного, но неподходящего, согласно субъективной оценке, ответа.

Инсайт как способ решения проблемы, поставленной при изучении креативного мышления, представляет парадоксальные моменты психологических механизмов творчества, так как эта внезапно возникающая идея решения проблемы как озарение признается единственно верной (что является характеристикой конвергентного мышления), хотя, с другой стороны, признанной основой 
творческого процесса является генерация множества идей как результат гибкого и разнообразного дивергентного мышления.

Хорошо известная четырехфазная модель творческого процесса, предложенная Г. Уоллесом, с логическим анализом постановки проблемы на его начальном этапе и критическим выбором наиболее подходящего варианта ответа в финальной фазе (см., например: Sadler-Smith, 2015) подтверждается результатами метаанализа публикаций, посвященных изучению нейрофизиологических коррелятов инсайта (Shen et al., 2018). Сделаны выводы о наличии интегрированной сети областей, активируемых инсайтом, которая включает правую медиальную лобную извилину, левую нижнюю лобную извилину, левую миндалину и правый гиппокамп, причем эти участки мозга в разной степени включались на протяжении четырех этапов решения проблемы. На стадии постановки задачи большая активация была отмечена для левой передней части поясной извилины, а период инкубации был представлен широко распределенной сетью структур в передней части мозга (ВА 6, 8, 44 и 47) и средней затылочной извилины (BА 19). В процессе озарения доминировала активность правого гиппокампа и левой миндалины, а верификация ответа была связана с функциями правой нижней части лобной извилины. Согласно заключению авторов другого обзора нейрофизиологических коррелятов креативности, вариативность структур мозга, включенных в поиск решения поставленной проблемы, определяется не только его временно́й динамикой, но и самим типом и сложностью задания и необходимыми для решения когнитивными ресурсами (Dietrich, Kanso, 2010).

Неоднородность процессов поиска оригинальной идеи отражена в других моделях творческого мышления. Согласно структурно-уровневому подходу, инсайт возникает вследствие смены доминирующих уровней мышления и передачи найденного решения с интуитивного уровня на логический (Пономарев, 1976), что отражается в особенностях распространения активации в семантической сети (Валуева, 2015). В ходе анализа динамики репрезентации проблемы и/или пространства поиска решения показано, что исходную репрезентацию проблемы определяют закономерности процессов восприятия и ресурсы знания, а последующие изменения обусловлены результативностью деятельности, для чего требуется последовательная реструктуризация пространства концептуального знания с расширением или ограничением поля поиска идей с использованием аналитических операторов (Öllinger et al., 2014).

Таким образом, результаты нейрофизиологических исследований инсайтного решения вербальных задач указывают на вовлечение нейронных сетей преимущественно височной коры и гиппокампа, выполняющих функции семантически организованной системы памяти. Остаются, однако, неясными механизмы генерации ассоциаций идей и их «весовой» значимости, определяющей передачу информации с интуитивного уровня на логический для уверенного осознания решения. Отмеченные выше ЭЭГ- и фМРТ-корреляты инсайта, представленные в префронтальной и цингулярной коре, которые выполняют функции выбора релевантной информации и контроля принятия 
решения (Aziz-Zadeh et al., 2009; Kizilirmak et al., 2016; Li et al., 2019), могут отражать финальную его фазу: признание верной возникшей в уме догадки. Критическое мышление как когнитивная стратегия, состоящая из последовательного логического рассуждения, анализа и проверки истинности возникающих идей на основе известных критериев, противопоставляется творческому вследствие внезапности и неосознанности инсайта как способа решения творческой задачи. Основными функциями критического мышления являются аргументированная селекция, оценка и контроль решения проблемы (Халперн, 2000). Вследствие обнаруженной связи функциональной активности префронтальной и цингулярной коры и с исполнительным контролем когнитивной деятельности, и с инсайтом возникает вопрос о причинах такого эффекта. Имеет смысл рассмотреть нейрофизиологические корреляты критического мышления как необходимого этапа творческой деятельности.

\section{Нейрофизиологические корреляты критического мышления при решении творческой задачи}

Предложенная Дж.П. Гилфордом модель интеллекта с выделением конвергентных и дивергентных ментальных операций, несмотря на критические замечания относительно ее психометрической ценности (Богоявленская, $2004)$, остается актуальной для нейрофизиологических исследований и сегодня, представляя сочетание разных уровней мышления, необходимое для постановки и/или решения новой проблемы (Разумникова, 2009б; Benedek et al., 2011, 2014; Fink, Benedek, 2014; Jauk et al., 2013; Razumnikova, 2000; Runco, Yoruk, 2014). Дивергентное мышление отражает способность к гибкому поиску разнообразных способов решения проблемы, каждое из которых может быть признано верным. «Необычное использование обычного предмета» - проблемная ситуация, позволяющая оценить количественный показатель генерации идей, оригинальность которых определяется частотой встречаемости или мнением эксперта. При отсутствии критического отношения к спонтанно возникающим вариантам решения такой задачи требуемая новизна и оригинальность вряд ли может быть достигнута.

Сочетание разных когнитивных операций в креативности заложено в моделях BVSR (Blind Variation and Selective Retention) (Campbell, 1960; Simonton, 2011, 2013) или «GENEPLORE» (Generate and Explore) (Finke et al., 1992). Поиск решения, согласно BVSR, влечет за собой как систематические, так и стохастические комбинаторные процедуры, которые включают избыточность «слепых» вариаций разнообразных ассоциаций, расфокусированное внимание с выборочным удержанием идей и эвристический поиск с использованием индивидуальных критериев идентификации ответа, согласно компетенциям в исследуемой предметной области. Выделяют исследовательскую и отборочную формы BVSR. Согласно модели GENEPLORE, творческий процесс мышления - это активный и целенаправленный процесс с повторяющимися состояниями с фазой генерации новой идеи на основе формирования ассоциаций следов памяти и исследованием этих идей с позиций возможного 
применения с учетом различного контекста. Подобный компонент - настойчивое исследование разных возможностей решения проблемы в сочетании с гибкой обработкой информации - заложен в модели креативности DPCM (Dual Pathway to Creativity model) (Nijstad et al., 2010). Таким образом, каждая из упомянутых моделей креативности наряду со спонтанным поиском идей «вширь» включает их аналитическую обработку «вглубь» и построение плана дальнейшего поиска, исходя из принимаемого решения о путях достижимости результата.

Интегральным психометрическим показателем успешности аналитического мышления и эффективности контроля ментальных операций можно считать IQ, именно поэтому достаточно много нейрофизиологических исследований посвящено изучению специфики регионарного взаимодействия между функциональными нейронными системами, включенными в контроль когнитивных операций (например: Разумникова, 2009б; Beaty et al., 2014b; Benedek et al., 2011, 2014; Santarnecchi et al., 2017). Имеются доказательства значимого вклада когнитивного контроля в показатели как интеллекта, так и креативности (Benedek et al., 2014; Chuderski, Necka, 2010; Chen et al., 2019; Chrysikou, 2019). Эти данные, а также положительные корреляции как флюидного, так и генерализованного компонентов интеллекта с показателями креативности (Beaty et al., 2014b; Cho et al., 2010; Jauk et al., 2013) при усилении этой связи после инструкции придумывать оригинальные решения, а не генерировать как можно больше разнообразных вариантов (Nusbaum, Silvia, 2011), указывают на важность когнитивного контроля в творческом мышлении.

Свидетельства в пользу общей для креативности и интеллекта функциональной системы с включением ее отдельных звеньев в зависимости от стратегии решения проблемы и ее специфики получены на основе данных о том, что связанная с флюидным интеллектом нейронная сеть объединяет заднюю систему внимания с передней системой различения значимости информации разной модальности (salience network) и левой фронто-париетальной системой контроля (Santarnecchi et al., 2017).

Однако анализ соотношения творческих способностей и интеллекта с применением теории управления сетью данных при структурной визуализации мозга, полученных с помощью диффузионного тензорного изображения (Kenett et al., 2018), выявил специфику нейрофизиологических коррелятов этих показателей. Оказалось, что интеллект связан со способностью смещать активность мозговой системы в легко достижимые состояния с функциональным центром в правой нижней теменной доле, а креативность - со способностью формировать сложные состояния за счет активности правой дорзолатеральной префронтальной коры при высоких интеграционных способностях сенсомоторных нейронных сетей и взаимодействии дефолтной и исполнительной систем мозга (DMN и ECN). Выше уже упоминалось об особенностях взаимодействия этих систем (Величковский и др., 2019; Beaty et al., 2014a, 2016), отражающих соотношение процессов спонтанной или целенаправленной селекции информации. Отметим, что продолжение фМРТ-исследований в этом направлении с привлечением выборки из 370 человек и анализом связей 
функций DMN и ECN с показателями вербальной креативности (тест Гилфорда «Необычное использование») под контролем вклада таких факторов, как пол, возраст и уровень IQ, выявило динамическую реконфигурацию этих нейронных сетей в покое, обусловленную функциональным включением в разные процессы памяти и внимания (Feng et al., 2019). Положительные корреляции с вербальной креативностью были значимы для показателей интеграции левых частей языковой и средней височной извилины в $\mathrm{DMN}$ и интеграции DMN и мозжечка, слуховой системы и лобно-теменной системы контроля, а также для двусторонней постцентральной извилины, принадлежащей сенсорной/соматомоторной сети. Обнаруженная регионарная специфика карт коннективности, согласно заключению авторов этого исследования, была связана с необходимостью обеспечения генерации креативных идей и выделения полезной информации.

Анализ соотношения разных функций когнитивного контроля с интеллектом и креативностью выявил положительный вклад функции обновления в уровень флюидного интеллекта и торможения совместно с обновлением - в креативность (Benedek et al., 2014). Эти функции представлены анатомически дорзолатеральной областью префронтальной коры и ассоциируются с показателем беглости, тогда как оригинальность связана с функцией переключения, представленной в ростральной части префронтальной коры (Chrysikou, 2019). Выбор оптимального решения является результатом гибкого переключения спонтанных процессов DMN и разнообразного сочетания функций исполнительного контроля селекции информации: гибкости внимания с переключением между разными альтернативами и снижения функциональной фиксированности вследствие ослабления тормозных процессов, что способствует генерации новых идей. Заключение о сочетании интернально сфокусированного внимания, целенаправленного извлечения информации из памяти и торможения доминантного, функционально фиксированного ответа как основных когнитивных процессах творческой деятельности сделано на основе анализа связанной с креативностью динамики взаимодействия нейронных сетей мозга (в том числе DMN, ECN и вентральной сети внимания) (Beaty et al., 2018).

Для поиска тех механизмов внимания, которые лежат в основе результативного решения поставленной креативной задачи, был выполнен ряд целенаправленных исследований, на результатах которых остановимся ниже.

\section{Особенности селекции информации при критическом мышлении или инсайте}

В качестве разных форм селекции информации, связанной с креативностью, рассматриваются механизмы обработки сенсорных потоков и их исполнительный контроль («bottom-up» и «top-down» соответственно), интернального и экстернального или «дефокусированного» и направленного внимания (Benedek et al., 2011; Fink, Benedek, 2014; Martindale, Hines, 1975). Перечисленные формы селекции информации объединяет противопоставление 
функций исполнительной системы когнитивного контроля и особенностей функциональной активации в сенсомоторной системе для выяснения закономерностей их взаимодействия. Так, согласно гипотезе «оппортунистической ассимиляции», новая идея возникает под воздействием триггера из окружающей среды (Seifert et al., 1995). С другой стороны, в качестве основы творческой идеи предлагается целенаправленная инициация новых комбинаций отдаленных ассоциаций (слов при использовании RAT или других вербальных заданий или образов при тестировании невербальной креативности) в процессе дивергентного мышления (Finke et al., 1992; Ansburg, Hill, 2003).

Согласно результатам анализа ЭЭГ-коррелятов креативности, возникла гипотеза, что успех в генерации оригинального ответа следует связать с «дефокусированным» вниманием, т.е. распределением функциональной активации в широко представленном семантическом пространстве или распределенных нейронных сетях (Разумникова, 2009a; Martindale, Hines, 1975). Действительно, серия исследований в этом направлении показала ассоциированное с креативностью снижение активации в лобных областях коры, согласно обнаруженному повышению синхронизации альфа-ритма (Benedek et al., 2011; Fink, Benedek, 2014; Razumnikova, 2007). Эффект повышения синхронизации альфа-осцилляций интерпретируется как показатель усиления интернального внимания, которое требуется для эффективного поиска оригинальной идеи с сопряженным ограничением ресурсов обработки экстернально поступающей информации (Fink, Benedek, 2014). В пользу гипотезы дефокусированного внимания рассматриваются результаты исследования инсайта с использованием китайских логогрифов, однако в этом случае повышение мощности альфа-ритма при успешной инсайтной стратегии было отмечено не в лобных, а в теменно-затылочных отделах коры (Cao et al., 2015). Генерализованное повышение альфа-колебаний предположительно отражает дефокусированную селекцию информации в широко распределенных нейронных сетях, представляющих семантическое пространство поиска новых идей. Вместе с этим для обозначения активированных на высоких частотах локально организованных нейронных ансамблей, которые оптимальным образом координируются (синхронизируются) для решения проблемы на фоне удаленного взаимодействия нейронных сетей, предложено понятие «дифференциальное внимание» (Petsche, 1996; Razumnikova, 2000).

Результаты других исследований указывают, что креативность скорее характеризуется эффективным переключением с дефокусированного внимания, которое необходимо для решения неявной, двойственной проблемы, к фокусированному в условиях четко очерченной задачи (Vartanian, 2009; Zabelina, Robinson, 2010). Показаны различия в ресурсах внимания у креативных и аналитических лиц: лучшее выполнение RAT сочеталось со способностью к диффузному вниманию (использованию периферической подсказки для решения анаграмм), тогда как эффективность решения дедуктивных заданий не была связана с этой способностью (Ansburg, Hill, 2003). При сопоставлении особенностей внимания и выполнения задания на составные отдаленные ассоциации установлено, что, если оно следует за выполнением другого 
задания, требующего концентрации внимания, участники исследования сообщали об использовании аналитического способа решения творческого задания, а после того, как они занимались быстрой идентификацией объекта, требующей внимания к широкому пространству и слабым ассоциациям, сообщали об использовании инсайта (Wegbreit et al., 2012). Более широкое поле селекции информации при дивергентном мышлении было показано и в другом исследовании, причем RAT в этом случае рассматривалась как задача, совмещающая дивергентный и аналитический подходы (Wronska et al., 2018).

Подобное объяснение поиска ассоциации с функциональным объединением процессов целенаправленного поддерживающего выполнение задания внимания с «дефокусированным» и «дифференцированным» было предложено для объяснения выявленного паттерна низкочастотных тета- и альфа1- и высокочастотных бета2-осцилляций при анализе ЭЭГ-коррелятов генерации отдаленных ассоциаций по сравнению с простыми ассоциативными цепочками (Razumnikova, 2007). Полученные результаты показали регионарно широко представленную активацию коры на частоте бета2-диапазона вместе с повышением мощности тета-ритма в лобных областях и десинхронизацией альфа1,2-ритма в задних отделах коры. Оригинальность ответов при выполнении RAT положительно коррелировала с повышением когерентности бета2-биопотенциалов фронто-париетальной коры и с повышением не только когерентности, но и мощности альфа1-ритма в височных и теменных областях левого полушария.

Инструкция мысленной оценки сгенерированной идеи не только сопровождается синхронизацией высокочастного альфа-ритма в лобных областях коры, но и усиливает этот эффект при генерации идеи, что рассматривается как повышение интернального внимания к репрезентации интернальной памяти (Hao et al., 2016). Таким образом, критическое мышление может служить стимулятором творческого решения проблемы, хотя, согласно описанному ЭЭГ-корреляту, не отличается от спонтанного поиска оригинальной идеи.

Известны положительные эффекты подсказки или переноса решения по аналогии и обучения креативности, однако реализуются они не всегда и даже могут иметь негативное влияние за счет фиксации на предложенных примеpax (Holyoak, Morrison, 2005; Osman, 2008). Торможение фиксационного эффекта, в свою очередь, оказывает положительное влияние на оригинальность решения креативной задачи (Agogué et al., 2014; Chrysikou et al., 2016). Исследование нейрофизиологической природы фиксации выявило регионарную специфику синхронизации альфа-биопотенциалов в лобных и височнотеменных областях коры с большим эффектом для лиц, способных находить отдаленные ассоциации (как полагают авторы, за счет интернально организованных сематических ассоциаций и селективных процессов) (Camarda et al., 2018).

Положительная связь способностей к дивергентному мышлению и когнитивного торможения обнаружена также с использованием показателей выполнения задач Струпа или Навона (Edl et al., 2014; Zabelina, Robinson, 
2010), в которых эффект торможения оценивается эффективностью разрешения конфликта между цветовым и семантическим значением стимула или его локальных и глобальных свойств. В этом случае речь идет об особенностях экстернального внимания, связанных с креативностью.

Анализ значения тормозного контроля в эффективности креативного мышления, выполненный с учетом фактора возраста, показал, что индивидуальная стратегия генерации креативной идеи представляет комбинацию принципиально разных когнитивных процессов с использованием ресурсов имплицитной или эксплицитной памяти и формированием фиксационного эффекта вследствие развития исполнительного контроля поведения в раннем онтогенезе или сохранности гибкого мышления даже на его поздних стадиях, позволяющего адаптироваться к новым условиям жизни (Разумникова, Николаева, 2019).

При изучении механизмов креативности особый интерес вызывают организация семантического пространства и закономерности такого извлечения информации, которые приводят к новому решению проблемы. В связи с этим задача использования предмета была рассмотрена с альтернативной оценкой «распространенное-необычное» и «пригодное-непригодное» (Rataj et al., 2018). Относительно большая активность теменно-затылочных участков коры правого полушария в высокочастотном альфа-диапазоне и передних отделов левого полушария по сравнению с правым в низкочастотном альфадиапазоне отмечена для альтернативного использования по сравнению с обычным. Эти изменения альфа-биопотенциалов при оценке альтернативы авторы связывают с бо́льшими требованиями к семантической обработке информации с созданием концептуальных повторных представлений и соответствующим повышением ресурсов внимания, о чем свидетельствует не только низкочастотная альфа-реактивность, но и большие амплитуды поздних компонентов вызванного потенциала.

Доказательства в пользу дуальной модели креативности (Nijstad et al., 2010) получены вследствие обнаруженной U-образной динамики мощности альфа-колебаний с ее повышением как на начальном этапе поиска идей с опорой на ассоциативное мышление и ресурсы памяти, так и при финальной их разработке и оценке на основе процессов исполнительного контроля (Rominger et al., 2019).

Таким образом, описанные особенности внимания (и их соответствующие экспериментальные доказательства) отражают необходимые для креативного выполнения задания компоненты селекции информации: либо критический перебор множества идей на основе сознательно актуализированных ресурсов семантической или эпизодической памяти, либо селекция информации осуществляется, не достигая сферы сознания. Структурами мозга, необходимыми для переключения разных стратегий селекции информации и выбора ответа при решении экспериментальных творческих задач в зависимости от их сложности и содержания, в первую очередь являются префронтальная и цингулярная области коры. Временна́я динамика и степень доминирования каждого компонента функциональной нейронной системы определяются не толь- 
ко условиями экспериментального задания, но и индивидуальной «преднастройкой» мозга для его решения. Поэтому столь значительное внимание уделяется в последнее время поиску закономерностей во взаимодействии исполнительной и дефолтной нейронных сетей мозга для объяснения механизмов творческой деятельности и стимуляции творческого потенциала (Величковский и др., 2019; Beaty et al., 2014a, 2015, 2016, 2018, 2019; de Pisapia et al., 2016; Feng et al., 2019; Ogawa et al., 2018; Shi et al., 2018; Sunavsky, Poppenk, 2020).

Можно заключить, что решение экспериментальной творческой задачи возможно на основе как инсайта, так и критического анализа идей, возникающих при поиске нестандартного ответа. ЭЭГ-коррелятом инсайта является синхронизация альфа-биопотенциалов, а регионарная специфика этого эффекта обусловлена особенностями креативного задания; например, височные и префронтальные области связываются с инсайтом при тестировании вербальной креативности. Результаты томографических исследований выявили ключевое значение паттернов «преднастройки» нейронных сетей покоя: дефолтной и исполнительной систем в лобных и теменных областях мозга, причем разные участки лобной коры связаны с разными показателями креативности. Гетерогенность творческого процесса отражается совмещением критического анализа возможных вариантов решения проблемы и инсайта. Структурами мозга, необходимыми для извлечения информации из памяти и гибкого использования разных стратегий селекции информации с целью выбора оригинального ответа при решении экспериментальных творческих задач, являются префронтальная и цингулярная области коры и гиппокамп. Включение в поиск решения проблемы противоположных когнитивных процессов - конвергентного или дивергентного мышления, направленного или дефокусированного и интернального или экстернального внимания - сопровождается временно́й и структурной реорганизацией активации или торможения в широко представленных нейронных системах мозга, регионарная специфика которых зависима от сложности и содержания заданий для тестирования креативности.

\section{Литература}

Бакулин, И. С., Пойдашева, А. Г., Медынцев, А. А., Лагода, Д. Ю., Кремнева, Е. И., Легостаева, Л. А., Синицын, Д. О., Супонева, Н. А., Пирадов, М. А. (2020). Нейробиологические основы инсайта (решения задач озарением). Успехи физиологических наук, 51(1), 72-86.

Богоявленская, Д. Б. (2004). Что выявляют тесты интеллекта и креативности? Психология. Журнал Высшей школы экономики, 1(2), 54-65.

Валуева, Е. А. (2015). Сигнальная модель инсайта: основные положения и соотношение с научными взглядами Я.А. Пономарева. Психологический журнал, 36(6), 35-44.

Величковский, Б. М., Князев, Г. Г., Валуева, Е. А., Ушаков, Д. В. (2019). Новые подходы в исследованиях творческого мышления: от феноменологии инсайта к объективным методам и нейросетевым моделям. Вопросы психологии, 3, 3-16. 
Пономарев, Я. А. (1976). Психология творчества. М.: Наука.

Психологический словарь. (2007). М.: ОЛМА ПРЕСС Образование.

Разумникова, О. М. (2009а). Особенности селекции информации при креативном мышлении. Психология. Журнал Высшей школы экономики, 6(3), 134-161.

Разумникова, О. М. (2009б). Связь частотно-пространственных параметров фоновой ЭЭГ с уровнем интеллекта и креативности. Журнал высшей нервной деятельности им. И.П. Павлова, 59(6), 686-695.

Разумникова, О. М., Ларина, Е. Н. (2005). Полушарные взаимодействия при поиске оригинальных вербальных ассоциаций: особенности когерентности биопотенциалов коры у креативных мужчин и женщин. Журнал высшей нервной деятельности им. И.П. Павлова, 6, 777-787.

Разумникова, О. М., Николаева, Е. И. (2019). Тормозные функции мозга и возрастные особенности организации когнитивной деятельности. Успехи физиологических наук, 1, 75-89.

Халперн, Д. (2000). Психология критического мьиления. СПб.: Питер.

Шелепин, К. Ю., Шелепин, Ю. Е. (2015). Нейрофизиология «инсайта». Петербургский психологический журнал, 11, 19-38.

Ссылки на зарубежные источники см. в разделе References после англоязычного блока.

Разумникова Ольга Михайловна - профессор, кафедра психологии и педагогики, Новосибирский государственный технический университет, доктор биологических наук. Сфера научных интересов: когнитивная психология, психология креативности и интеллекта, дифференциальная психофизиология.

Контакты: razoum@mail.ru

\title{
Neurophysiological Mechanisms of Solution of Experimental Creative Problems: Insight or/and Critical Analysis?
}

O.M. Razumnikova

${ }^{a}$ Novosibirsk State Technical University, 20 K. Marks avenue, Novosibirsk, 630073, Russian Federation

\begin{abstract}
The literature review examines different points of view on the processes of interaction of neural networks of the brain in selection of information and decision-making on the basis of insight or a critical analysis of the ideas generated during creativity testing. The BVSR (Blind Variation and Selective Retention) and "GENEPLORE" (Generate and Explore) models have been proposed to study the combination of different cognitive operations in creativity. The analysis of the insight in experimental studies was performed using tasks on heuristic thinking, perception of dual figures, the tests of remote associations (RAT) or compound remote associations (CRA). The information on the most stable EEG correlate of insight is given, which is the synchronization of alpha biopotentials and changes in the intensity and regional specificity of this effect, due to the characteristics of the creative task. The results of tomographic studies revealed the key importance of specific patterns of "pre-setting" of neural networks at rest: default and executive systems and the dynamics of the interaction of the frontal and parietal regions of the brain,
\end{abstract}


reflecting different indicators of creativity. The role of a critical analysis in the solution of a problem and the importance of inhibitory functions in the selection of relevant information are modulating the creative process. Due to the inclusion of opposing processes in the search for an original solution to the problem - of convergent or divergent thinking, directed or defocused and internal or external attention - there is a temporary and structural reorganization of activation or inhibition in the neural systems of the brain. The brain structures required for switching between different strategies of information selection and for selecting an answer when solving experimental creative tasks depending on their complexity and content are the prefrontal and cingular cortical areas, and the hippocampus.

Keywords: creativity, insight, critical thinking, information selection, inhibitory functions, neural networks, electroencephalography, tomography.

\section{References}

Agogué, M., Kazakci, A., \& Hachuel, A. (2014). The impact of type of examples on originality: Explaining fixation and stimulation effects. The Journal of Creative Behavior, 48(1), 1-12. https://doi.org/10.1002/jocb.37

Ansburg, P. I., \& Hill, K. (2003). Creative and analytic thinkers differ in their use of attentional resources. Personality and Individual Differences, 34(7), 1141-1152. https://doi.org/10.1016/S0191-8869(02)00104-6

Aziz-Zadeh, L., Kaplan, J. T., \& Iacoboni, M. (2009). "Aha!”: The neural correlates of verbal insight solutions. Human Brain Mapping, 30(3), 908-916. https://doi.org/10.1002/hbm.20554

Bakulin, I. S., Poydasheva, A. G., Medyntsev, A. A., Lagoda, D. Yu., Kremneva, E. I., Legostaeva, L. A., Sinitsyn, D. O., Suponeva, N. A., \& Piradov, M. A. (2020). Neurobiological principles of insight problem solving. Uspekhi Fiziologicheskih Nauk [Advances of Physiological Sciences], 51(1), 72-86. https://doi.org/10.31857/S0301179820010038 (in Russian)

Beaty, R. E., Benedek, M., Silvia, P. J, \& Schacter, D. L. (2016). Creative cognition and brain network dynamics. Trends in Cognitive Sciences, 20(2), 87-95.

Beaty, R. E., Benedek, M., Wilkins, R. W., Jauk, E., Fink, A., Silvia, P. J., Hodges, D. A., Koschutnig, K., \& Neubauer, A. C. (2014a). Creativity and the default network: A functional connectivity analysis of the creative brain at rest. Neuropsychologia, 64, 92-98. https://doi.org/10.1016/j.neuropsychologia.2014.09.019

Beaty, R. E., Kenett, Y. N., Christensen, A. P., Rosenberg, M. D., Benedek, M., Chen, Q., Fink, A., Qiu, J., Kwapil, T. R., Kane, M. J., \& Silvia, P. J. (2018). Network neuroscience of creative cognition: mapping cognitive mechanisms and individual differences in the creative brain. Proceedings of the National Academy of Sciences of the United States of America, 115(5), 1087-1092. https://doi.org/10.1073/pnas.1713532115

Beaty, R. E., Silvia, P. J., Nusbaum, E. C., Jauk, E., \& Benedek, M. (2014b). The roles of associative and executive processes in creative cognition. Memory $\mathcal{E}$ Cognition, 42(7), 1-12.

Beaty, R., Benedek, M., Kaufman, S. B., \& Silvia, P. J. (2015). Default and executive network coupling supports creative idea production. Scientific Reports, 5, Article 10964. https://doi.org/10.1038/srep10964

Beaty, R.E., Seli, P., \& Schacter, D. L. (2019). Network neuroscience of creative cognition: mapping cognitive mechanisms and individual differences in the creative brain. Current Opinion in Behavioral Sciences, 27, 22-30. https://doi.org/10.1016/j.cobeha.2018.08.013 
Beeman, M. J., \& Bowden, E. M. (2000). The right hemisphere maintains solution related activation for yetto-be-solved problems. Memory \& Cognition, 28, 1231-1241. https://doi.org/10.3758/BF03211823

Benedek, M., Bergner, S., Könen, T., Fink, A., \& Neubauer, A. C. (2011). EEG alpha synchronization is related to top-down processing in convergent and divergent thinking. Neuropsychologia, 49, 3505-3511.

Benedek, M., Jauk, E., Sommer, M., Arendasy, M., \& Neubauer, A. C. (2014). Intelligence, creativity, and cognitive control: The common and differential involvement of executive functions in intelligence and creativity. Intelligence, 46, 73-83.

Bogoyavlenskaya, D. B. (2004). What do tests of intelligence and creativity show? Psychology.Journal of the Higher School of Economics, 1(2), 54-65. (in Russian)

Camarda, A., Salvia É., Vidal, J., Weil B., Poirel, N., \& Houdéa, O. (2018). Neural basis of functional fixedness during creative idea generation: an EEG study. Neuropsychologia, 118(Pt. A), 4-12. https://doi.org/10.1016/j.neuropsychologia.2018.03.009

Campbell, D. T. (1960). Blind variation and selective retention in creative thought as in other knowledge processes. Psychological Revier, 67, 380-400.

Cao, Z., Li, Y., Hitchman, G., Qiu, J., \& Zhang, Q. (2015). Neural correlates underlying insight problem solving: Evidence from EEG alpha oscillations. Experimental Brain Research, 233, 2497-2506. https://doi.org/10.1007/s00221-015-4338-1

Chen, Y., Spagna, A., Wu, T. Kim, T. H., Wu, Q., Chen, C., Wu, Y., \& Fan, J. (2019). Testing a cognitive control model of human intelligence. Scientific Reports, 9, Article 2898. https://doi.org/10.1038/s41598-019-39685-2

Cho, S. H., Nijenhuis, J. T., van Vianen, A. E., Kim, H.-B., \& Lee, K. H. (2010). The relationship between diverse components of intelligence and creativity. Journal of Creative Behavior, 44(2), 125-137. https://doi.org/10.1002/j.2162-6057.2010.tb01329.x

Chrysikou, E. G. (2019). Creativity in and out of (cognitive) control. Current Opinion in Behavioral Sciences, 27, 94-99. https://doi.org/10.1016/j.cobeha.2018.09.014

Chrysikou, E. G., Motyka, K., Nigro, C., Yang, S. I., \& Thompson-Schill, S. L. (2016). Functional fixedness in creative thinking tasks depends on stimulus modality. Psychology of Aesthetics, Creativity, and the Arts, 10(4), 425-435. https://doi.org/10.1037/aca0000050

Chuderski, A., \& Necka, E. (2010). Intelligence and cognitive control. In A. Gruszka, G. Matthiews, \& D. Szymura (Eds.), Handbook of individual differences in cognition: Attention, memory, and executive control (pp. 263-282). Springer Verlag. https://doi.org/10.1007/978-1-4419-1210-7_16

Dandan, T., Haixue, Z., Wenfu, L., Wenjing, Y., Jiang, Q., \& Qinglin, Z. (2013). Brain activity in using heuristic prototype to solve insightful problems. Behavioural Brain Research, 253, 139-144. https://doi.org/10.1016/j.bbr.2013.07.017

De Pisapia, N., Bacci, F., Parrott, D., \& Melcher, D. (2016). Brain networks for visual creativity: a functional connectivity study of planning a visual artwork. Scientific Reports, 6, Article 39185. https://doi.org/10.1038/srep39185

Dietrich, A. (2004). The cognitive neuroscience of creativity. Psychonomic Bulletin \& Reviere, 11, 1011026. https://doi.org/10.3758/BF03196731

Dietrich, A., \& Kanso, R. (2010). A review of EEG, ERP, and neuroimaging studies of creativity and insight. Psychological Bulletin, 136(5), 822-848. https://doi.org/10.1037/a0019749

Edl, S., Benedek, M., Papousek, I., Weiss, E. M., \& Fink, A. (2014). Creativity and the Stroop interference effect. Personality and Individual Differences, 69, 38-42. https://doi.org/10.1016/j.paid.2014.05.009 
Feng, Q., He, L., Yang, W., Zhang, Y., Wum, X., \& Qiu, J. (2019). Verbal creativity is correlated with the dynamic reconfiguration of brain networks in the resting state. Frontiers in Psychology, 10, 894. https://doi.org/10.3389/fpsyg.2019.00894

Fink, A., \& Benedek, M. (2014). EEG alpha power and creative ideation. Neuroscience \& Biobehavioral Reviews, 44(100), 111-123. https://doi.org/10.1016/j.neubiorev.2012.12.002

Finke, R. A., Ward, T. B., \& Smith, S. M. (1992). Creative cognition: theory, research and applications. Cambridge: The MIT Press.

Fleck, J., \& Weisberg, R. W. (2013). Insight versus analysis: Evidence for diverse methods in problem solving. Journal of Cognitive Psychology, 25(4), 436-463. https://doi.org/10.1080/20445911.2013.779248

Hao, N., Ku, Y., Liu, M., Hu, Y., Bodner, M., Grabner, R.H., \& Fink, A. (2016). Reflection enhances creativity: Beneficial effects of idea evaluation on idea generation. Brain and Cognition, 103, 30-37. https://doi.org/10.1016/j.bandc.2016.01.005

Holyoak, K. J., \& Morrison, R. G. (2005). Thinking and reasoning: A reader's guide. In K. Holyoak \& B. Morrison (Eds.), The Cambridge handbook of thinking and reasoning (pp. 1-9). Cambridge University Press.

Jauk, E., Benedek, M., Dunst, B., \& Neubauer, A. C. (2013). The relationship between intelligence and creativity: New support for the threshold hypothesis by means of empirical breakpoint detection. Intelligence, 41(4), 212-221.

Jung-Beeman, M., Bowden, E. M., Haberman, J., Frymiare, J. L., Arambel-Liu, S., Greenblatt, R., Reber, P. J., \& Kounios, J. (2004). Neural activity when people solve verbal problems with insight. PLoS Biology, 2(4), 500-510. https://doi.org/10.1371/journal.pbio.0020097

Kenett, Y. N., Medaglia, J. D., Beaty, R. E., Chen, Q., Betzel, R. F., Thompson-Schill, S. L., \& Qiu, J. (2018). Driving the brain towards creativity and intelligence: A network control theory analysis. Neuropsychologia, 118(Pt. A), 79-90. https://doi.org/10.1016/j.neuropsychologia.2018.01.001

Khalil, R., Godde, B., \& Karim, A. A. (2019). The link between creativity, cognition, and creative drives and underlying neural mechanisms. Frontiers in Neural Circuits, 13, 18. https://doi.org/10.3389/fncir.2019.00018

Khalpern, D. (2000). Psikhologiya kriticheskogo myshleniya [The psychology of critical thinking]. Saint Petersburg: Piter. Original work published 1997.

Kizilirmak, J. M., Thuerich, H., Folta-Schoofs, K., Schott, B. H., \& Richardson-Klavehn, A. (2016). Neural correlates of learning from induced insight: A case for reward-based episodic encoding. Frontiers in Psychology, 7, 1693. https://doi.org/10.3389/fpsyg.2016.01693

Kounios, J., \& Beeman, M. (2014). The cognitive neuroscience of insight. Annual Review of Psychology, 65, 71-93. https://doi.org/10.1146/annurev-psych-010213-115154

Laukkonen, R. E., \& Tangen, J. M. (2018). How to detect insight moments in problem solving experiments. Frontiers in Psychology, 9, 282. https://doi.org/10.3389/fpsyg.2018.00282

Lawson, A. E. (2010). Basic inferences of scientific reasoning, argumentation, and discovery. Science Education, 94(2), 336-364. https://doi.org/10.1002/sce.20357

Li, W., Li, G., Ji, B., Zhang, Q., \& Qiu, J. (2019). Neuroanatomical correlates of creativity: Evidence from voxel-based morphometry. Frontiers in Psychology, 10, 155. https://doi.org/10.3389/fpsyg.2019.00155

Martindale, C., \& Hines, D. (1975). Creativity and cortical activation during creative, intellectual and EEG feedback tasks. Biological Psychology, 3(2), 91-94. https://doi.org/10.1016/03010511(75)90011-3 
Mednick, S. A. (1962). The associative basis of the creative process. Psychological Revier, 69(3), 220232. https://doi.org/10.1037/h0048850

Mednick, S. A., \& Mednick, M. (1971). Remote associates test: Examiner's manual. Houghton Mifflin.

Nijstad, B. A., De Dreu, C. K. W., Rietzschel, E., \& Baas, M. (2010). The dual pathway to creativity model: Creative ideation as a function of flexibility and persistence. European Review of Social Psychology, 21(1), 34-77. https://doi.org/10.1080/10463281003765323

Nusbaum, E. C., \& Silvia, P. J. (2011). Are intelligence and creativity really so different? Fluid intelligence, executive processes, and strategy use in divergent thinking. Intelligence, 39(1), 36-45. https://doi.org/10.1016/j.intell.2010.11.002

Ogawa, T., Aihara, T., Shimokawa, T., \& Yamashita, O. (2018). Large-scale brain network associated with creative insight: combined voxel-based morphometry and resting-state functional connectivity analyses. Scientific Reports, 8(1), Article 6477. https://doi.org/10.1038/s41598-018-24981-0

Öllinger, M., Jones, G., \& Knoblich, G. (2014). The dynamics of search, impasse, and representational change provide a coherent explanation of difficulty in the Nine-Dot problem. Psychological Research, 78(2), 266-275. https://doi.org/10.1007/s00426-013-0494-8

Osman, M. (2008). Positive transfer and negative transfer/antilearning of problem-solving skills. Journal of Experimental Psychology: General, 137(1), 97-115. https://doi.org/10.1037/0096-3445.137.1.97

Petsche, H. (1996). Approaches to verbal, visual and musical creativity by EEG coherence analysis. International Journal of Psychophysiology, 24(1-2), 145-159. https://doi.org/10.1016/s01678760(96)00050-5

Ponomarev, Ya. A. (1976). Psikhologiya tvorchestva [The psychology of creativity]. Moscow: Nauka.

Psikhologicheskii slovar' [Psychological Dictionary]. (2007). Moscow: OLMA PRESS Obrazovanie.

Qiu, J., Li, H., Jou, J., Liu, J., Luo, Y., \& Feng, T. (2010). Neural correlates of the Aha experiences: evidence from an fMRI study of insight problem solving. Cortex, 46(3), 397-403. https://doi.org/10.1016/j.cortex.2009.06.006

Rataj, K., Nazareth, D. S., \& van der Velde, F. (2018). Use a spoon as a spade? Changes in the upper and lower alpha bands in evaluating alternate object use. Frontiers in Psychology, 9, 1941. https://doi.org/10.3389/fpsyg.2018.01941

Razumnikova, O. M. (2000). Functional organization of different brain areas during convergent and divergent thinking: an EEG investigation. Cognitive Brain Research, 10(1-2), 11-18. https://doi.org/10.1016/s0926-6410(00)00017-3

Razumnikova, O. M. (2007). Creativity related cortex activity in the remote associates task. Brain Research Bulletin, 73, 96-102.

Razumnikova, O. M. (2009a). Peculiarities of information selection in the process of creative thinking. Psychology. Journal of the Higher School of Economics, 6(3), 134-161. (in Russian)

Razumnikova, O. M. (2009b). The relationship between frequency-spatial parameters of the baseline EEG and levels of intelligence and creativity. Zhurnal Vysshei Neronoi Deyatel'nosti im. I.P. Pavlova, 59(6), 686-695. (in Russian)

Razumnikova, O. M., \& Larina, E. N. (2005). Hemispheric interactions during a search of original verbal associations: EEG coherence in creative men and women. Zhurnal Vysshei Neronoi Deyatel'nosti im. I.P. Pavlova, 6, 777-787. (in Russian)

Razumnikova, O. M., Nikolaeva, E. I. (2019). Inhibitory brain functions and age-associated specificities in organization of cognitive activity Uspekhi Fiziologicheskikh Nauk, 1, 75-89. (in Russian)

Rominger, C., Papousek, I., Perchtold, C.M., Benedek, M., Weiss, E.M., Schwerdtfeger, A., \& Fink, A. (2019). Creativity is associated with a characteristic U-shaped function of alpha power changes 
accompanied by an early increase in functional coupling. Cognitive, Affective, \& Behavioral Neuroscience, 19, 1012-1021. https://doi.org/10.3758/s13415-019-00699-y

Rothmaler, K., Nigbur, R., \& Ivanova, G. (2017). New insights into insight: Neurophysiological correlates of the difference between the intrinsic "aha" and the extrinsic "oh yes" moment. Neuropsychologia, 95, 204-214. https://doi.org/10.1016/j.neuropsychologia.2016.12.017

Runco, M. A., \& Yoruk, S. (2014). The neuroscience of divergent thinking. Activitas Nervosa Superior, 56, 1-16. https://doi.org/10.1007/BF03379602

Sadler-Smith, E. (2015). Wallas' four-stage model of the creative process: More than meets the eye? Creativity Research Journal, 27(4), 342-352. https://doi.org/10.1080/10400419.2015.1087277

Santarnecchi, E., Emmendorfera, A., \& Pascual-Leonea, A. (2017). Dissecting the parieto-frontal correlates of fluid intelligence: A comprehensive ALE meta-analysis study. Intelligence, 63, 9-28.

Seifert, C. M., Meyer, D. E., Davidson, N., Patalano, A. L., \& Yaniv, I. (1995). Demystification of cognitive insight: Opportunistic assimilation and the prepared-mind perspective. In R. J. Sternberg \& J. E. Davidson (Eds.), The nature of insight (pp. 65-124). Cambridge, MA: The MIT Press.

Shelepin, K. Yu., \& Shelepin, Yu. E. (2015). Neurophysiology of the insight. Peterburgskii Psikhologicheskii Zhurnal, 11, 19-38. (in Russian)

Shen, W., Tong, Y., Li, F., Yuan, Y., Hommel, B., Liu, C., \& Luo, J. (2018). Tracking the neurodynamics of insight: A meta-analysis of neuroimaging studies. Biological Psychology, 138, 189-198. https://doi.org/10.1016/j.biopsycho.2018.08.018

Shen, W., Yuan, Y., Liu, C. \& Luo, J. (2017). The roles of the temporal lobe in creative insight: An integrated review. Thinking and Reasoning, 23(4), 321-375. https://doi.org/10.1080/13546783.2017.1308885

Shi, L., Beaty, R. E., Chen, Q., Sun, J., Wei, D., Yang, W., \& Qiu, J. (2020). Brain entropy is associated with divergent thinking. Cerebral Cortex, 30(2), 708-717. https://doi.org/10.1093/cercor/bhz120

Shi, L., Sun, J., Xia, Y., Ren, Z., Chen, Q., Wei, D., Yang, W., \& Qiu, J. (2018). Large-scale brain network connectivity underlying creativity in resting-state and task fMRI: Cooperation between default network and frontal-parietal network. Biological Psychology, 135, 102-111. https://doi.org/10.1016/j.biopsycho.2018.03.005

Simonton, D. K. (2011). Creativity and discovery as blind variation: Campbell's (1960) BVSR Model after the half-century mark. Review of General Psychology, 15(2), 158-174. https://doi.org/10.1037/a0022912

Simonton, D. K. (2013). Creative problem solving as sequential BVSR: Exploration (total ignorance) versus elimination (informed guess). Thinking Skills and Creativity, 8, 1-10. https://doi.org/10.1016/j.tsc.2012.12.001

Smith, K. A., Huber, D. E., \& Vul, E. (2013). Multiply-constrained semantic search in the Remote Associates Test. Cognition, 128(1), 64-75. https://doi.org/10.1016/j.cognition.2013.03.001

Sprugnoli, G., Rossi, S., Emmendorfer, A., Rossi, A., Liew, S.-L., Tatti, E., di Lorenzo, G., PascualLeone, A., \& Santarnecchi, E. (2017). Neural correlates of Eureka moment. Intelligence, 62, 99118. https://doi.org/10.1016/j.intell.2017.03.004

Sunavsky, A., \& Poppenk, J. (2020). Neuroimaging predictors of creativity in healthy adults. NeuroImage, 206, Article 116292, https://doi.org/10.1016/j.neuroimage.2019.116292

Valueva, E. A. (2015). Insight-as-signal model: Main assumptions and relation to Ya.A. Ponomarev's conception. Psikhologicheskii Zhurnal, 36(6), 35-44. (in Russian)

Vartanian, O. (2009). Variable attention facilitates creative problem solving. Psychology of Aesthetics, Creativity, and the Arts, 3, 57-59. 
Velichkovskii, B. M., Knyazev, G. G., Valueva, E. A., \& Ushakov, D. V. (2019). New approaches in studies of creative thinking: From phenomenology of insight to objective methods and neuronetwork models. Voprosy Psikhologii, 3, 3-16. (in Russian)

Virtue, S., Parrish, T., \& Jung-Beeman, M. (2008). Inferences during story comprehension: Cortical recruitment affected by predictability of events and working memory capacity.Journal of Cognitive Neuroscience, 20(12), 2274-2284. https://doi.org/10.1162/jocn.2008.20160

Webb, M. E., Little, D. R., \& Cropper, S. J. (2016). Insight is not in the problem: Investigating insight in problem solving across task types. Frontiers in Psychology, 7, 1424. https://doi.org/10.3389/fpsyg.2016.01424

Wechsler, S. M., Saiz, C., Rivas, S. F., Vendramini, C. M. M., Almeid, L. S., Mundim, M. C., \& Franco, A. (2018). Creative and critical thinking: Independent or overlapping components? Thinking Skills and Creativity, 27, 114-122.

Wegbreit, E., Suzuki, S., Grabowecky, M., Kounios, J., \& Beeman, M. (2012). Visual attention modulates insight versus analytic solving of verbal problems. Journal of Problem Solving, 4(2), 94-115.

Wronska, M. K., Kolańczyk, A., \& Nijstad, B. A. (2018). Engaging in creativity broadens attentional scope. Frontiers in Psychology, 9, 1772. https://doi.org/10.3389/fpsyg.2018.01772

Zabelina, D., \& Robinson, M. D. (2010). Creativity as flexible cognitive control. Psychology of Aesthetics Creativity and the Arts, 4(3), 136-143. https://doi.org/10.1037/a0017379

Zhang, M., Tian, F., Wu, X., Liao, S., \& Qiu, J. (2011). The neural correlates of insight in Chinese verbal problems: An event related-potential study. Brain Research Bulletin, 84, 210-214. https://doi.org/10.1016/j.brainresbull.2011.01.001

Zhao, Q., Zhou, Z., Xu, H., Fan, W., \& Han, L. (2014). Neural pathway in the right hemisphere underlies verbal insight problem solving. Neuroscience, 256, 334-341. https://doi.org/10.1016/j.neuroscience.2013.10.019

Olga M. Razumnikova - Professor, Department of Psychology and Pedagogics, Novosibirsk State Technical University, DSc in Biology.

Research Area: cognitive psychology, psychology of creativity and intelligence, differential psychophysiology.

E-mail: razoum@mail.ru 